\title{
Evertor muscle fatigue decreases the passive inversion joint position sense in female volleyball players
}

\author{
DOI: https://doi.org/10.5114/pq.2021.105750
}

\author{
Hisham Mohamed Hussien ${ }^{1,2} \oplus$, Alaa Samir Mohamed², Abeer AbdelRahman Yamany² \\ ${ }^{1}$ Physical Therapy Department, College of Applied Medical Sciences, University of Hail, Ha'il, Saudi Arabia \\ ${ }^{2}$ Basic Science Department, Faculty of Physical Therapy, Cairo University, Giza, Egypt
}

\begin{abstract}
Introduction. The study aimed to investigate the effect of evertor muscle fatigue on passive inversion joint position sense in female volleyball players.

Methods. A cross-sectional design was implemented in this study. The passive inversion joint position sense of 25 college-age female volleyball players was assessed in fatigue and non-fatigue conditions. A $10^{\circ}$ subtalar inversion was used as a target angle for position sense evaluation. The assessment of position sense and the induction of fatigue were performed with a Biodex System isokinetic device. The average error of joint position sense was measured in both fatigue and non-fatigue conditions. Results. There was a significant reduction in passive inversion joint position sense when the evertor muscles were fatigued $(p<0.001)$.

Conclusions. Peroneal muscle fatigue declines the subtalar joint inversion position sense in female volleyball players.

Key words: evertors, fatigue, isokinetic, peroneal muscles, proprioception
\end{abstract}

\section{Introduction}

Fatigue is considered one of the most popular complaints. It can be experienced by diseased, normal, or athletic populations [1]. Muscular fatigue arises when there is a decline in skeletal muscle performance during physical activity [2, 3]. In sport, fatigue commonly occurs because of prolonged training and excessive stress coming from repeated competitions. Athletes usually experience fatigue near the end of their training sessions or competitive matches.

Volleyball players, especially females, are exposed to fatigue during their practice [4]. They demonstrate a high rate of lateral ankle sprains (LASs) [5, 6]. These injuries occur near the end of the matches, when muscular fatigue usually takes place [7, 8]. Activities such as spiking, blocking, and changing direction place severe stress on the ankle joint and the surrounding protective tissues, such as ligaments and muscles [9].

If ignored, fatigue could lead to overwork, chronic fatigue syndrome, overtraining syndrome, and, consequently, premature retirement [1]. The characteristics of the skeletal muscles change with fatigue [10]. For example, fatigue has been shown to impair the mechanical proprieties of the involved muscle [11], with the potential result of altering the function of the muscular proprioceptors (muscle spindles) [12]. Signals from these receptors are important for proper reaction timing, better reporting of sense of movement and position, and producing optimal postural reflexes [13].

Evertor muscles play an important protective role; they contract eccentrically to prevent excessive inversion movement in the subtalar joint and protect the lateral ankle ligaments from injury. Their fatigue affects the function of their proprioceptors, increasing the risk of falling and decreasing the ability of skeletal muscles to counteract excessive stress. Therefore, the subject becomes vulnerable to lateral ankle injuries [14].
A better understanding of fatigue and of how it affects different body functions can play a major role in enhancing the performance of female volleyball players and decreasing the risk of fatigue-related injuries in this sport [12]. Studying the influence of evertor muscle fatigue on joint position sense in female volleyball players may provide evidence concerning the role of fatigue as a risk factor for LASs [15]. Consequently, appropriate measures could be considered to control the harmful effect of fatigue and decrease the risk for LASs in female volleyball players.

Unfortunately, the literature contains few studies that have investigated the influence of fatigue on the ankle joint proprioception. The effect of evertor fatigue on ankle joint position sense has been investigated on 4 occasions [16-19]; 3 studies have focused solely on normal subjects [16, 18, 19], while only one addressed male soccer players [17]. All of them except one [19] have reported a negative effect of evertor muscle fatigue on the accuracy of ankle proprioception. These papers, however, did not mention the encountered limitations and did not consider volleyball players.

The purpose of this study was to examine the effect of evertor muscle fatigue on passive inversion joint position sense (PIJPS) in female volleyball players.

\section{Subjects and methods}

Design

This cross-sectional study was performed in an isokinetic laboratory setting.

\section{Sample size}

A pilot study was conducted and its results were used to calculate the appropriate sample size for the main study.

Correspondence address: Hisham Mohamed Hussein, Physical Therapy Department, College of Applied Medical Sciences, University of Hail, P.O. Box: 2240, Ha'il, Saudi Arabia, e-mail: hm.hussein@uoh.edu.sa, https://orcid.org/0000-0003-2184-6147 
Five volleyball players who did not participate in the main study joined the pilot study. PIJPS was measured with an isokinetic dynamometer during fatigue and non-fatigue conditions of the evertor muscles of the dominant limb. Average error [8] was calculated around $10^{\circ}$ inversion. The $\mathrm{G}^{*} \mathrm{P}$ ower software (University of Düsseldorf, Düsseldorf, Germany) was used with the following data: power of $80 \%$, significance level of $5 \%$, effect size of 0.6 . The results indicated that 25 participants would be required to execute the study.

\section{Participants}

A total of 25 healthy college-age female volleyball players participated in this study. Healthy females who had practised volleyball regularly for the previous year were eligible. The recruitment process was conducted within a campus of Cairo University, Egypt, through announcements using students' social media groups, between August and November 2018

Participants with a history of diabetes, chronic ankle instability, rheumatologic disease, restricted joint mobility, lower extremity injury or surgery, neurological disorders, as well as those using medications that could alter deep sensation were excluded.

\section{Instrumentation}

A Biodex System 3 Pro isokinetic dynamometer (Biodex Medical Systems, Shirley, NY, USA) was used to assess PIJPS and induce fatigue to the evertor muscles.

The isokinetic machine has been widely used to produce muscular fatigue in research [16-19]. Fatigue has been also evoked by other methods, such as prolonged static activity [20] and actual sports activities [17]. The isokinetic dynamometer has been reported to be a safe, valid, and reliable tool for inducing fatigue and assessing joint position sense [21]. It was used in the study because of its validity and reliability for assessing muscle performance [22-24], measuring joint position sense [25], as well as inducing and assessing fatigue [21].

\section{Procedures}

The study procedures and participants' rights were explained before the start of the study; then, the recruited subjects were screened for inclusion and exclusion criteria. Their personal and demographic data were recorded (Table 1).

As a warm-up, each participant was instructed to perform stretching for ankle muscles and hamstrings [18].

\section{Proprioception assessment}

The participants were instructed to sit on the Biodex isokinetic chair. The back support of the chair was inclined backward to form a $60^{\circ}$ angle with the horizontal plane to relax the hamstring muscle [19].

The participant was asked to rest the dominant lower limb on a horizontally aligned limb support pad and place the tested foot on the footplate [19]. Dominance was determined by establishing the preferred leg used to kick a ball [26].

Straps on the lap and knee were used to secure the subject's position. The participants were asked to fold their arms across the chest, let the non-tested foot hang freely, and close their eyes to eliminate visual feedback (Figure 1).

The chosen protocol allowed each participant to perform 3 familiarization trials before conducting the actual test. The

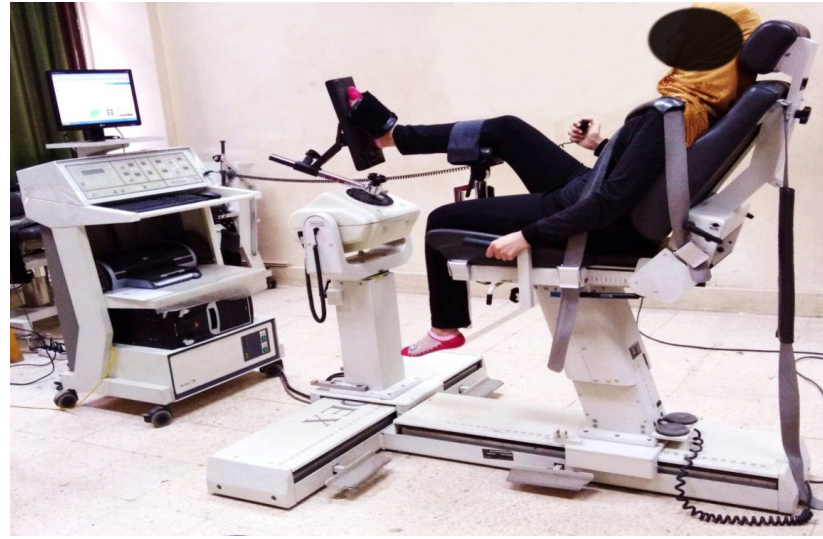

Figure 1. Assessment of passive inversion joint position sense

dynamometer was adjusted to move the tested foot in an inversion with a speed of $2 \%$ s $[16,18]$. When the dynamometer reached $10^{\circ}$ of inversion, it sustained this position for 5 seconds [16]. Meanwhile, the participant was instructed to memorize this position. The tested foot then was returned to the starting position to perform the actual test trial. In the actual test, the dynamometer moved the tested foot in the inversion direction slowly and the participant was instructed to push a stop-button upon reaching the target angle [18]. Once the participant pushed the button, the dynamometer stopped and the device recorded the inversion angle in degrees. This test was repeated 3 times and the average error around the predetermined angle was calculated and used for the subsequent data analysis.

\section{Fatigue protocol}

After recording the joint position sense, the participants were allowed to rest for 5 minutes before a fatiguing protocol was implemented [17]. The tested foot was secured to the foot attachment of the isokinetic device in the anatomical position. The knee was flexed at $45^{\circ}$ and the hip at $70^{\circ}$. The foot and thigh were secured in place with 2 Velcro straps. Three maximal isometric eversion efforts, with 5-minute rest intervals, were performed to determine the participant's maximum voluntary contraction torque, later applied to establish the onset of fatigue.

To induce fatigue, the participant was instructed to perform repeated eversion contractions above $70 \%$ of the predetermined maximum voluntary contraction. This testing protocol had revealed valid results in a previous study [27]. The test was terminated if the participant reached the fa-

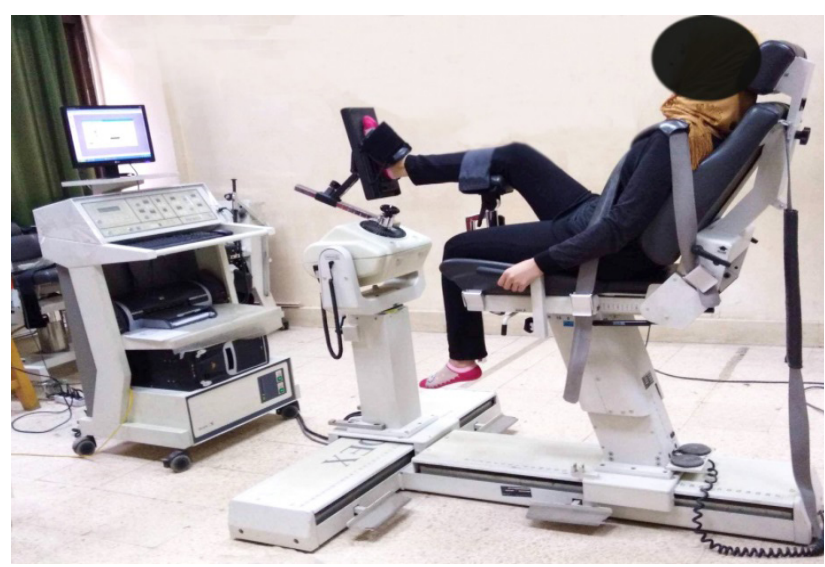

Figure 2. Induction of fatigue to evertor muscles by using the isokinetic machine 
tigue level. Fatigue occurred when 3 successive contractions dropped below $50 \%$ of the predetermined maximum level. The reassessment of joint position sense was performed within 2 minutes of the end of the fatigue protocol before recovery took place [17] (Figure 2).

\section{Statistical analysis}

Data analysis was performed with the SPSS version 24 software (SPSS Inc., Chicago, USA). Numerical data were summarized by using means, standard deviations, median, and range. A paired $t$-test was performed to compare the average error values in the fatigued and non-fatigued conditions. The alpha level was set at $p<0.05$.

\section{Ethical approval}

The research related to human use has complied with all the relevant national regulations and institutional policies, has followed the tenets of the Declaration of Helsinki, and has been approved by the Cairo University Research Ethics Committee (No.: P.T. REC/012/001928).

\section{Informed consent}

Informed consent has been obtained from all individuals included in this study.

\section{Results}

All participants were young adults. Their age ranged from 18 to 24 years. Their mean body mass index remained within the normal category (Table 1$)$. Only 2 participants (8\%) demonstrated left leg dominance, while 23 (92\%) had a dominant right lower limb.

The statistical analysis demonstrated that PIJPS was significantly higher during the fatigue condition at $10^{\circ}$ of ankle inversion $(p<0.001)$ (Table 2$)$.

\section{Discussion}

The current study investigated the influence of fatigue of the evertor muscles on subtalar joint PIJPS. To select the appropriate sample size, a pilot study was conducted using the target angle of interest ( $10^{\circ}$ of inversion). The results showed a decline in the ability to recognize the target angle when evertor muscles were fatigued.

The influence of fatigue on inversion position sense was studied before in healthy subjects $[16,18]$. Despite fatigue is a common problem in sports, its effect on the athletic pop-

Table 1. The general characteristics of participants

\begin{tabular}{|l|c|c|c|c|}
\hline Characteristics & Mean \pm SD & Median & Minimum & Maximum \\
\hline Age (years) & $20 \pm 2$ & 20 & 18 & 24 \\
\hline Weight $(\mathrm{kg})$ & $61 \pm 6$ & 60 & 50 & 71 \\
\hline Height $(\mathrm{cm})$ & $163 \pm 5$ & 164 & 151 & 170 \\
\hline $\begin{array}{l}\text { Body mass index } \\
\left(\mathrm{kg} / \mathrm{m}^{2}\right)\end{array}$ & $22.8 \pm 1.6$ & 23.1 & 18.4 & 24.8 \\
\hline
\end{tabular}

Table 2. Comparison between the average errors of PIJPS at $10^{\circ}$ inversion before and after evertor muscle fatigue

ulation has been investigated once among male soccer players [17].

The present study has the following unique characteristics: it is among the few studies that induced fatigue to the peroneal muscles and measured position sense in the inversion range, it is the second study conducted in competitive sports players, it is the first to target female volleyball players, and the calculation of the sample size might increase the generalizability of the result.

The results of the current study agree with those of multiple previous studies [16-18], all of which have reported impairments in joint position sense in fatigue conditions.

Mohammadi and Roozdar [17] assessed joint position sense at $15^{\circ}$ and maximum minus $5^{\circ}$ in inversion after inducing fatigue among male soccer players. The authors divided the recruited sample into 2 groups and used different techniques to induce fatigue in each group. Passive and active joint position sense were assessed before and during fatigue. Position sense errors were higher during fatigue in both groups.

Sandrey and Kent [18] reported that joint position sense was affected more around higher angles $\left(20^{\circ}\right)$ when compared with lower angles $\left(5^{\circ}\right)$. A similar finding was observed earlier [28].

Lin et al. [16] compared the joint position sense between a group of healthy individuals and others who had a history of an ankle injury. They found that fatigue, as well as an ankle injury, led to higher errors around $10^{\circ}$ of ankle inversion when compared with $20^{\circ}$.

Contrary to the current results, South and George [19] concluded that fatigue did not affect joint position sense. They studied the effect of evertor muscle fatigue on position sense around different angles in inversion and eversion. However, their study concerned mainly normal non-athletic subjects. Moreover, they did not address gender variations in the response to fatigue.

Evertor muscles of the ankle have a special ability to produce force. This ability comes from the long distance traversed by the peroneus longus tendon [29]. The eccentric force of the evertors can control inversion stress and constitute a protective mechanism against LASs [30].

This impairment in joint position sense increases sagittal plane joint displacement, as well as reduces muscle power, joint stability, and muscular reaction time to neural impulses [21]. Fatigue increases these changes in joint position sense and raises the risk of LASs in volleyball players [31].

The relationship between fatigue and joint position sense is still unclear in the literature [17]. However, the muscle spindles may play a role. It is now generally accepted that muscle spindles are an important source of position sense information [32]. According to many reports, they are easily affected by the development of fatigue inside the muscle [32-34]. Therefore, one can attribute the decline in joint position sense to the inhibition of muscle spindle function during fatigue.

The current study is of particular importance for females practising volleyball. According to the results, fatigue that occurs to evertors of the subtalar joint may contribute to the de-

\begin{tabular}{|c|c|c|c|c|c|c|c|}
\hline & \multirow{2}{*}{$\begin{array}{r}\text { Non-fatigued } \\
(\text { mean } \pm S D)\end{array}$} & \multirow{2}{*}{$\begin{array}{c}\text { Fatigued } \\
(\text { mean } \pm S D)\end{array}$} & \multirow{2}{*}{ MD } & \multicolumn{2}{|c|}{$95 \% \mathrm{Cl}$ of $\mathrm{MD}$} & \multirow{2}{*}{$\%$ of change } & \multirow{2}{*}{$p$} \\
\hline & & & & Lower & Upper & & \\
\hline PIJPS around $10^{\circ}$ inversion & $1.12 \pm 0.47^{\circ}$ & $1.64 \pm 0.63^{\circ}$ & $0.51^{\circ}$ & $0.30^{\circ}$ & $0.73^{\circ}$ & $45.5 \%$ & $<0.001$ \\
\hline
\end{tabular}


crease of joint position sense and increase the risk for LASs, usually seen in females playing volleyball.

\section{Limitations}

Although sample size calculation was performed before the study, the small number of participants may limit the generalizability of the results.

\section{Conclusions}

Evertor muscle fatigue may affect the PIJPS of the subtalar joint and increase the risk of LASs.

\section{Disclosure statement}

No author has any financial interest or received any financial benefit from this research.

\section{Conflict of interest}

The authors state no conflict of interest.

\section{References}

1. Wan J-J, Qin Z, Wang P-Y, Sun Y, Liu X. Muscle fatigue: general understanding and treatment. Exp Mol Med. 2017;49(10):e384; doi: 10.1038/emm.2017.194.

2. García-Manso JM, Rodríguez-Ruiz D, Rodríguez-Matoso D, de Saa Y, Sarmiento S, Quiroga M. Assessment of muscle fatigue after an ultra-endurance triathlon using tensiomyography (TMG). J Sports Sci. 2011;29(6): 619-625; doi: 10.1080/02640414.2010.548822.

3. El Gohary TM, Ibrahim SR, El-din Mahmoud WS. Effect of muscle length on fatigue induced by low frequency current stimulation in human medial gastrocnemius muscle. Int J Ther Rehabil Res. 2016;5(4):158-165; doi: 10.5455/ijtrr.000000158.

4. Doherty C, Delahunt E, Caulfield B, Hertel J, Ryan J, Bleakley $\mathrm{C}$. The incidence and prevalence of ankle sprain injury: a systematic review and meta-analysis of prospective epidemiological studies. Sports Med. 2014;44(1): 123-140; doi: 10.1007/s40279-013-0102-5.

5. Roos KG, Kerr ZY, Mauntel TC, Djoko A, Dompier TP, Wikstrom EA. The epidemiology of lateral ligament complex ankle sprains in National Collegiate Athletic Association sports. Am J Sports Med. 2017;45(1):201-209; doi: 10.1177/0363546516660980.

6. Feger MA, Glaviano NR, Donovan L, Hart JM, Saliba SA, Park JS, et al. Current trends in the management of lateral ankle sprain in the United States. Clin J Sport Med. 2017;27(2):145-152; doi: 10.1097/JSM.000000000000 0321.

7. Kilic O, Maas M, Verhagen E, Zwerver J, Gouttebarge V. Incidence, aetiology and prevention of musculoskeletal injuries in volleyball: a systematic review of the literature. Eur J Sport Sci. 2017;17(6):765-793; doi: 10.1080/17 461391.2017.1306114.

8. Márquez G, Alegre LM, Jaén D, Martin-Casado L, Aguado $X$. Sex differences in kinetic and neuromuscular control during jumping and landing. J Musculoskelet Neuronal Interact. 2017;17(1):409-416.

9. Hadzic V, Sattler T, Topole E, Jarnovic Z, Burger H, Dervisevic E. Risk factors for ankle sprain in volleyball players: a preliminary analysis. Isokinet Exerc Sci. 2009; 17(3):155-160; doi: 10.3233/IES-2009-0347.

10. Rey E, Corredoira FJ, Costa PB, Pérez-Ferreirós A, Fernández-Villarino MA. Acute effects of training load on contractile properties during a competitive microcycle in elite soccer players. Biol Sport. 2020;37(2):157-163; doi: 10.5114/biolsport.2020.93041.

11. Bilodeau M, Henderson TK, Nolta BE, Pursley PJ, Sandfort GL. Effect of aging on fatigue characteristics of elbow flexor muscles during sustained submaximal contraction. J Appl Physiol. 2001;91(6):2654-2664; doi: 10.1152/ jappl.2001.91.6.2654.

12. Bisson EJ, McEwen D, Lajoie Y, Bilodeau M. Effects of ankle and hip muscle fatigue on postural sway and attentional demands during unipedal stance. Gait Posture. 2011;33(1):83-87; doi:10.1016/j.gaitpost.2010.10.001.

13. Hussien HM, Abdel-Raoof NA, Kattabei OM, Ahmed HH. Effect of Mulligan concept lumbar SNAG on chronic nonspecific low back pain. J Chiropr Med. 2017;16(2):94102; doi: 10.1016/j.jcm.2017.01.003.

14. Aman JE, Elangovan N, Yeh I-L, Konczak J. The effectiveness of proprioceptive training for improving motor function: a systematic review. Front Hum Neurosci. 2015; 8:1075; doi: 10.3389/fnhum.2014.01075.

15. Wilson EL, Madigan ML. Effects of fatigue and gender on peroneal reflexes elicited by sudden ankle inversion. J Electromyogr Kinesiol. 2007;17(2):160-166; doi: 10.1016/ j.jelekin.2006.01.009.

16. Lin YH, Li CW, Tsai LY, Liing R-J. The effects of muscle fatigue and proprioceptive deficits on the passive joint senses of ankle inversion and eversion. Isokinet Exerc Sci. 2008;16(2):101-105; doi: 10.3233/IES-2008-0303.

17. Mohammadi F, Roozdar A. Effects of fatigue due to contraction of evertor muscles on the ankle joint position sense in male soccer players. Am J Sports Med. 2010; 38(4):824-828; doi: 10.1177/0363546509354056.

18. Sandrey MA, Kent TE. The effects of eversion fatigue on frontal plane joint position sense in the ankle. J Sport Rehabil. 2008;17(3):257-268; doi: 10.1123/jsr.17.3.257.

19. South M, George KP. The effect of peroneal muscle fatigue on ankle joint position sense. Phys Ther Sport. 2007; 8(2):82-87; doi: 10.1016/j.ptsp.2006.12.001.

20. Caron O. Effects of local fatigue of the lower limbs on postural control and postural stability in standing posture. Neurosci Lett. 2003;340(2):83-86; doi: 10.1016/ s0304-3940(02)01455-6.

21. Yaggie JA, McGregor SJ. Effects of isokinetic ankle fatigue on the maintenance of balance and postural limits. Arch Phys Med Rehabil. 2002;83(2):224-228; doi: 10.1053/apmr.2002.28032.

22. Farrag A, Elsayed W. Habitual use of high-heeled shoes affects isokinetic soleus strength more than gastrocnemius in healthy young females. Foot Ankle Int. 2016; 37(9):1008-1016; doi: 10.1177/1071100716649172.

23. Coratella G, Beato M, Cè E, Scurati R, Milanese C, Schena $F$, et al. Effects of in-season enhanced negative workbased vs traditional weight training on change of direction and hamstrings-to-quadriceps ratio in soccer players. Biol Sport. 2019;36(3):241-248; doi: 10.5114/biolsport. 2019.87045.

24. Staniszewski M, Mastalerz A, Urbanik C. Effect of a strength or hypertrophy training protocol, each performed using two different modes of resistance, on biomechanical, biochemical and anthropometric parameters. Biol Sport. 2020;37(1):85-91; doi: 10.5114/biolsport.2020. 92517.

25. Drouin JM, Valovich-McLeod TC, Shultz SJ, Gansneder BM, Perrin DH. Reliability and validity of the Biodex System 3 Pro isokinetic dynamometer velocity, torque and position measurements. Eur J Appl Physiol. 2004;91(1): 22-29; doi: 10.1007/s00421-003-0933-0. 
26. Van Melick N, Meddeler BM, Hoogeboom TJ, Nijhuisvan der Sanden MWG, van Cingel REH. How to determine leg dominance: the agreement between self-reported and observed performance in healthy adults. PLoS One. 2017;12(12):e0189876; doi: 10.1371/journal.pone. 0189876.

27. Forestier N, Teasdale N, Nougier V. Alteration of the position sense at the ankle induced by muscular fatigue in humans. Med Sci Sports Exerc. 2002;34(1):117-122; doi: 10.1097/00005768-200201000-00018.

28. Boyle J, Negus V. Joint position sense in the recurrently sprained ankle. Aust J Physiother. 1998;44(3):159-163; doi: 10.1016/s0004-9514(14)60375-5.

29. Decourcy Hallinan JTP, Wang W, Pathria MN, Smitaman E, Huang BK. The peroneus longus muscle and tendon: a review of its anatomy and pathology. Skeletal Radiol. 2019;48(9):1329-1344; doi: 10.1007/s00256019-3168-9.

30. Hertel J. Functional anatomy, pathomechanics, and pathophysiology of lateral ankle instability. J Athl Train. 2002;37(4):364-375.

31. Kobayashi T, Tanaka M, Shida M. Intrinsic risk factors of lateral ankle sprain: a systematic review and meta-analysis. Sports Health. 2016;8(2):190-193; doi: 10.1177/19 41738115623775.

32. Hiemstra LA, Lo IK, Fowler PJ. Effect of fatigue on knee proprioception: implications for dynamic stabilization. J Orthop Sports Phys Ther. 2001;31(10):598-605; doi: 10.2519/jospt.2001.31.10.598.

33. Proske U. Exercise, fatigue and proprioception: a retrospective. Exp Brain Res. 2019;237(10):2447-2459; doi: 10.1007/s00221-019-05634-8.

34. Changela PK, Selvamani K, Ramaprabhu. A study to evaluate the effect of fatigue on knee joint proprioception and balance in healthy individuals. Med Sport. 2012;8(2): 1851-1857. 